\title{
Modeling a Two-Stage High-Power Anode Layer Thruster and its Plume
}

\author{
Michael Keidar,,- Yongjun Choi,,$\stackrel{ \pm}{a}$ and Iain D. Boyd $\stackrel{ \pm}{ \pm}$ \\ University of Michigan, Ann Arbor, Michigan 48109
}

DOI: $10.2514 / 1.22185$

\begin{abstract}
A model of a two-stage thruster with anode layer is developed based on a two-dimensional hydrodynamic approach. Each stage is considered separately, while the solution of the first stage provides the boundary conditions for the second stage. Current-voltage characteristic of the discharge in the first stage is calculated and is found to be in agreement with experiment. In the acceleration channel the plasma-wall interactions are studied and expansion of the high-voltage sheath near the acceleration channel wall is investigated. It is predicted that under the typical conditions the sheath expands significantly and the quasi-neutral plasma region is confined in the middle of the channel. For instance, in the case of a $3 \mathrm{kV}$ discharge voltage, the sheath thickness is about $1 \mathrm{~cm}$, which is a significant portion of the channel width. It is shown that near-wall sheath expansion leads to a shorter acceleration region. Wall erosion by the energetic ions is calculated. The erosion profiles and the total erosion rate generally agree well with available experimental data. Plasma flow analysis provides the boundary conditions for the plume expansion study. The plume model is partially validated by comparison of the ion current density distribution with experimental data.
\end{abstract}

\section{Nomenclature}

$A$

$C_{s}$

$d_{a}$

$E_{z}$

$I_{a}$

$j_{d}$

$j_{e}$

$j_{e}^{\text {th }}$

$m_{i}$

$N_{s}$

$n$

$n_{a}$

$Q_{\text {ion }}$

$Q_{j}$

$Q_{w}$

$s$

$T_{e}$

$U$

$U_{a}$

$U_{d}$

$U_{i}$

V

$V_{s}$

$\beta^{s}$

$\Delta \varphi_{w}$

$\varepsilon$

$v_{\text {ef }}$

$v_{w}=$

$i, a, e$

$=$

sou

$=$ a

$=$

$=$

$=$

$=$

$=$

$=$

$=$

$=$

$=$

$=$

$=$

$=$

$=$

$=$

$=$ ion

$=$

$=$

$=$

$=$

$=$
$=$ effective electron collision frequency

$=$ frequency of electron collisions with walls

$=$ respectively

Received 2 January 2006; revision received 29 May 2006; accepted for publication 27 July 2006. Copyright (C) 2006 by the American Institute of Aeronautics and Astronautics, Inc. All rights reserved. Copies of this paper may be made for personal or internal use, on condition that the copier pay the $\$ 10.00$ per-copy fee to the Copyright Clearance Center, Inc., 222 Rosewood Drive, Danvers, MA 01923; include the code 0748-4658/07 \$10.00 in correspondence with the CCC.

*Research Scientist, Department of Aerospace Engineering; keidar@ umich.edu. Senior Member AIAA.

${ }^{\dagger}$ Graduate Student, Department of Aerospace Engineering.

†Professor, Department of Aerospace Engineering. Associate Fellow AIAA.

\section{Introduction}

$\mathbf{H}$ ALL thrusters are among the most advanced and efficient types of electrostatic propulsion devices. The electric field in the Hall thruster is perpendicular to the magnetic field, and thus passing the electron current across a magnetic field leads to an electron closed drift or Hall drift that provides the efficient gas ionization. In the Hall thruster the particle acceleration takes place in a quasi-neutral plasma and thus is not limited by space charge effects unlike the ion thruster counterpart. The original idea of ion acceleration in crossed fields by using a magnetron-type configuration was first introduced by Zharinov [1,2] Since the initial development of this idea in the 1960s, numerous experimental and theoretical investigations have been conducted [3-10]

In a thruster with anode layer (TAL), the ion acceleration takes place over a thin layer near the anode with a length of about the electron Larmor radius. This is why the term "anode layer" was attributed to the Hall thruster with metal walls [1-10]. In this thruster, an electric discharge in the $E \times B$ fields is created in the short gap between magnetic poles where closed electron drift takes place. In this magnetized layer, the neutral atoms are ionized and accelerated.

Recently an interest in development of high-power Hall thrusters was renewed due to the fact that TAL technology has wide technical capabilities and range of parameters [11-13]. In Hall thrusters, a substantial fraction of the ions is directed toward the channel wall. The channel wall limits the discharge in the radial direction and protects the magnetic poles from erosion. Ion interaction with walls leads to wear of these walls due to ion bombardment and results in a shortened lifetime of such accelerators. Because reduced erosion of various thruster components, such as electrodes, insulators, screens, etc., is critical for the long term operation of the thruster, the TAL variant of Hall thruster technology seems to be beneficial because it has a very small acceleration region and therefore small area in contact with ions of materials where possible erosion occurs. Recently, a two-stage TAL that was developed more than 25 years ago by TsNIIMASH was presented [12]. This thruster using bismuth as the propellant demonstrated increasing efficiency with operating voltage through $8 \mathrm{kV}$ and specific impulse in the range of 2000$5000 \mathrm{~s}$ at power levels of 10-34 kW. Having many advantages, bismuth was found to be a suitable option as a propellant. Bismuth has low ionization potential, is heavy, and significantly less toxic than mercury, while much cheaper than xenon [12]. A detailed comparison between various propellant options for Hall thrusters was recently performed [14]. It was concluded that bismuth has some superior properties for high thrust, low specific impulse missions. 
Bismuth has advantages such as low cost, low ionization potentials, and lower sputter erosion.

It was demonstrated that TALs can operate in both single and twostage regimes; in the two-stage regime, ionization and acceleration take place in two separate discharges, so that at the exit plane of the first stage, the plasma is highly ionized and additional ionization in the second stage is insignificant [12]. It was demonstrated that the two-stage regime of operation has many advantages, for instance, the possibility to achieve much higher specific impulse [15]. When a two-stage thruster is considered, the most important region in terms of long time operation is the second acceleration stage in which a very large voltage drop is applied. Although, theoretically, discharges in both stages are separate, there is a coupling in which the second stage discharge affects the first stage discharge. Below some low discharge voltage at the first stage limit, which is about $250 \mathrm{~V}$, the discharge in the first stage cannot be sustained without the second stage [16]. In this mode the discharge is diffuse and it was found that plume divergence is minimal [16]. Thus, this discharge mode is preferable for a two-stage thruster.

Recently it was shown that an important aspect of high-power TAL's is the sheath formation near the channel wall [17]. Typically in a TAL, the channel walls have potential, which is equal to the cathode potential, leading to a significant potential drop between the wall and the plasma. As a result, a high-voltage space charge sheath is formed and the sheath thickness can be comparable to the channel width. Therefore, a transition between quasi-neutral and space charge regimes can occur in the case of a high voltage in the second stage [17]. This transition can have a significant effect on the TAL operation and therefore is extremely important for practical applications.

In this paper various aspects of the two-stage high-power bismuth TAL are studied. Models of plasma flow in the first and second stages are developed based on a 2-D hydrodynamic approach. In the second stage, plasma-wall interactions are studied in detail. In particular, erosion of the channel wall due to energetic ion impact is analyzed. Plasma flow analysis in the thruster provides boundary conditions for the plume simulation. Plasma plume expansion under various conditions is investigated. The overall model is validated by comparison with existing experimental data.

\section{Model of TAL Plasma Flow}

The considered thruster has a two-stage configuration to separate the ion production and acceleration zones. Previously, a model of the second acceleration stage was developed [17]. Recent efforts are concentrated on development of the first ionization stage. To this end, a 2-D hydrodynamic model of the first stage of the VHITAL160 is developed. The diffuse form of the discharge in the first stage is studied. This form of the discharge is sustained by the acceleration stage according to previous experiments. Therefore the model of the first stage is coupled with the acceleration stage [17] and initially electron temperature is used as a downstream boundary condition.

In this section, we consider the plasma flow, which starts at the anode of the first stage. Quasi neutrality is assumed and therefore the plasma presheath-sheath interface is considered as the lateral boundary for the plasma flow region. This model was described in detail elsewhere [18]. Here we just briefly review the main points of the model. We employ a hydrodynamic model in a 2-D domain assuming that the system reaches a steady state as shown in Fig. 1. A radial component of the magnetic field only is considered. The momentum and mass conservation equations for electrons, ions, and neutrals under such conditions have the following form:

$$
\begin{gathered}
n m_{i}\left(\boldsymbol{V}_{i} \nabla\right) \boldsymbol{V}_{i}=n e \boldsymbol{E}-\nabla P_{i}-\beta n m_{i} n_{a}\left(\boldsymbol{V}_{i}-\boldsymbol{V}_{a}\right) \\
\nabla \cdot\left(\boldsymbol{V}_{i} n\right)=\beta n n_{a} \\
\nabla \cdot\left(\boldsymbol{V}_{a} n_{a}\right)=-\beta n n_{a}
\end{gathered}
$$

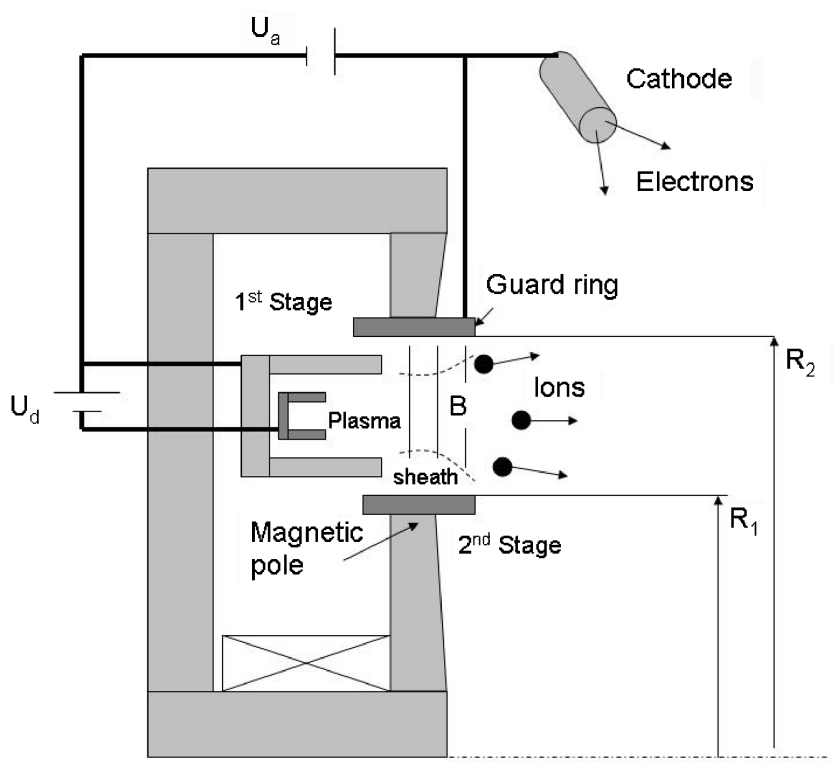

Fig. 1 Schematics of the thruster and computational domain.

$$
\begin{gathered}
0=-e n\left(\boldsymbol{E}+\boldsymbol{V}_{e} \times \boldsymbol{B}\right)-\nabla P_{e}-n v_{\mathrm{ef}} m_{e} \boldsymbol{V}_{e} \\
\frac{3}{2} \partial\left(j_{e} T_{e}\right) / \partial z=Q_{j}-Q_{w}-Q_{\text {ion }}
\end{gathered}
$$

where $Q_{j}=j_{e} E$ is the joule heat, $Q_{w}=v_{w} n\left(2 e T_{e}+e \Delta \varphi_{w}\right)$ represents the electron energy losses to the walls, and $Q_{\text {ion }}=$ $e n_{a} n U_{i} \beta$ represents ionization energy losses. The ionization rate is taken from [19]. To simplify the problem without missing the major physical effects, we consider one-dimensional flow of the neutrals, that is, $V_{a}=V_{\mathrm{az}}$. Because of a large potential drop across the sheath (see Sec. IV) it is assumed that the current flows only in the axial direction and current density is constant along the channel. Finally, electron transport is considered in a one-dimensional framework along the channel median. Because only the radial magnetic field component is considered, the electron transport is much greater in the azimuthal direction $(\boldsymbol{E} \times \boldsymbol{B}$ drift) than in the axial direction (drift diffusion due to collisions). We consider that electron transport across the magnetic field is due to several collision mechanisms: electron-neutral collisions and anomalous (Bohm) diffusion: $v_{\text {ef }}=v_{\text {en }}+v_{B}$, where $v_{\text {ef }}$ is the effective electron collision frequency. It was recognized long ago that including the classical mechanism of collisions cannot explain the electron transport observed in a Hall thruster [20]. However, there is no consensus about which of the possible mechanisms of electron transport is most significant in the Hall thruster [20]. In the TAL, which does not have significant secondary electron emission, anomalous transport due to plasma turbulence may be important. In the present work we will include anomalous (Bohm) transport [21]. The effective electron collision frequency related to the anomalous transport (Bohm diffusion) can be estimated as $v_{B}=\alpha \omega_{e}$, where $\alpha \sim 1 / 16$ is the Bohm empirical parameter in the classical formulation. The best fit with the experimental data of the potential drop for a given discharge current corresponds to $\alpha \sim 1 / 100$ instead of the classical value $\sim 1 / 16$. It should be noted that the conclusion derived by different authors was that the best fit with experiment corresponds to $\alpha \sim 1 / 80$ to $1 / 100([17,18,20])$.

The model includes bismuth evaporation and current continuity analysis at the anode. The near anode sheath is considered for which the potential drop is

$$
\Delta \varphi_{a}=\ln \left(\frac{j_{d}+e n_{e a} C_{s}}{j_{e}^{\text {th }}}\right)
$$

The discharge current in the range of 4-6 A is considered. The simulations correspond to the following case: mass flow rate is 
$10 \mathrm{mg} / \mathrm{s}$, magnetic field is $0.15 \mathrm{~T}$, and acceleration voltage is 2$5 \mathrm{kV}$.

To obtain a solution of the system of Eqs. (1-5) the following boundary conditions must be specified. At the downstream boundary (thruster exit plane) we specify an electron temperature of $T_{e}=$ $10 \mathrm{eV} \mathrm{[18].} \mathrm{The} \mathrm{plasma} \mathrm{density} \mathrm{at} \mathrm{the} \mathrm{entrance} \mathrm{to} \mathrm{the} \mathrm{second} \mathrm{stage} \mathrm{is}$ determined by the ionization rate in the first stage. Boundary conditions for ion velocity and density at the walls are determined by considering a plasma-sheath transition as described elsewhere [17].

The numerical analysis is similar to that developed previously [22]. We use the implicit two-layer method to solve the system of Eqs. (1-5). These equations are approximated by a two-layer six point scheme. The electron temperature distribution is calculated by iteration initially assuming a trial temperature distribution that satisfies the boundary conditions.

\section{Analysis of the Ionization Stage in a Two-Stage TAL}

In this section, we describe results of the computation of the plasma flow and electric characteristics of the discharge in the first ionization stage of the two-stage TAL. In particular, the ionization processes are studied in detail in the model of the first stage. The following input parameters are used to set up the problem: mass flow rate, discharge voltage, thruster geometry, and magnetic field distribution. Electron temperature calculated in the acceleration stage is used as a boundary condition. In the case of a $3 \mathrm{kV}$ acceleration stage voltage, the electron temperature at the interface between the two stages is about $18.5 \mathrm{eV}$. In the ionization stage, the energy equation [Eq. (5)] is simplified and a simple linear dependence of the electron temperature on the discharge voltage is used: $T_{e}=\beta U_{d}$. According to previous models of the anode layer, it can be assumed that $\beta=0.1$ [23]. Thus a higher discharge voltage will lead to a higher electron temperature in the ionization region.

The ionization fraction distribution in the ionization layer is shown in Fig. 2 with the discharge voltage as a parameter. Plasma density $n_{e a}$ at the anode was assumed to be 0.01 . It was found that initial plasma density does not affect the ionization process significantly. Higher discharge voltage leads to higher electron temperature that in turn affects the ionization rate. As a result, one can see that the higher discharge voltage causes a higher ionization degree near the anode. The ionization fraction sharply increases near the anode at a distance of about a millimeter and then saturates at the level dependent on the discharge voltage.

The two-dimensional density distribution in the first stage is shown in Fig. 3. It can be seen that the plasma density peaks near the anode due to strong ionization and then decreases due to acceleration of the ions along the channel.

One of the most important parameters of the discharge is the current-voltage characteristic. The calculated characteristic for the first stage is shown in Fig. 4. According to the model prediction, the

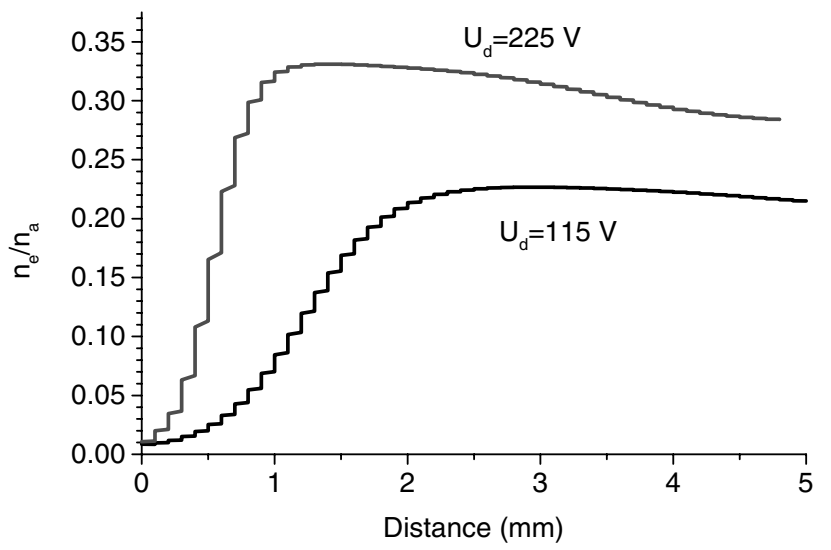

Fig. 2 Ionization fraction in the ionization layer near the anode with discharge voltage as a parameter.

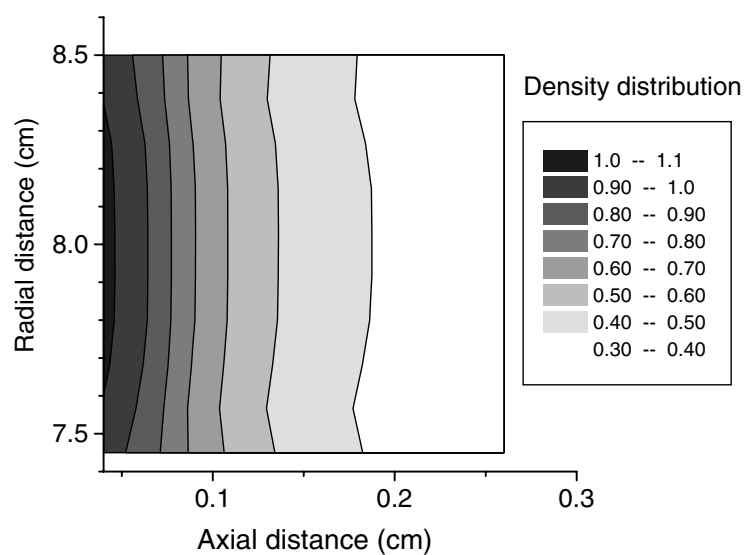

Fig. 3 Density distribution in the first stage. Discharge voltage: $140 \mathrm{~V}$.

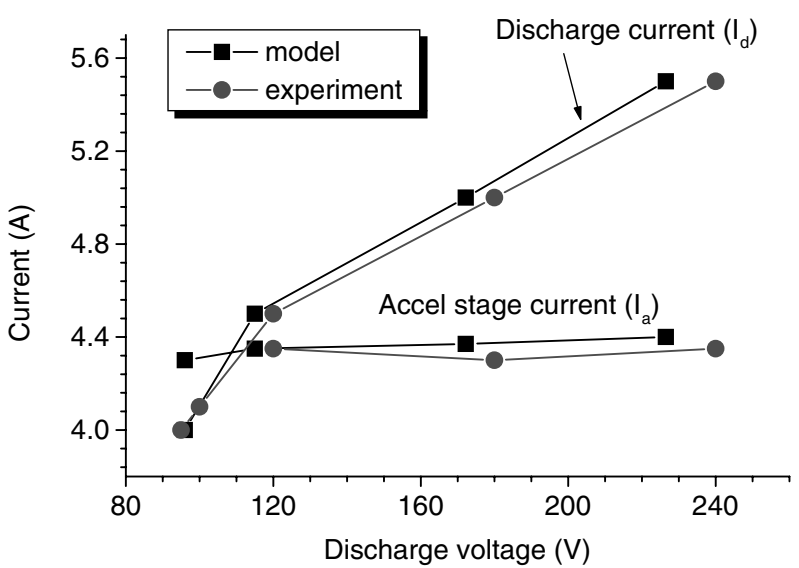

Fig. 4 Current-voltage characteristics of the first stage and dependence of the acceleration stage current on the discharge voltage in the first stage. Comparison with experimental data. Experimental data were taken from [16].

discharge current increases with the discharge voltage. Higher discharge current is supported by increased ionization as shown above. The typical experimental characteristic is also shown in Fig. 4. It can be seen that the thruster has an optimal discharge characteristic in a narrow range of discharge voltage. This optimal range of the discharge voltage is calculated here. Higher discharge voltage (above $250 \mathrm{~V}$ ) leads to very high electron temperature in the first stage and causes significant increase of the discharge current as well as the acceleration stage current. The discharge current in the first stage increases with the voltage, while the discharge current in the second stage is fairly constant. The discharge current increases due to ionization increase in the vicinity of the anode as shown in Fig. 2. On the other hand, the plasma density at the exit of the first stage is fairly independent of the discharge voltage thus leading to flat dependence of the acceleration stage current on the discharge voltage. One can see that our model predictions are in good agreement with experiment.

\section{Analysis of the Acceleration Stage}

The solution of the plasma flow in the first stage provides the boundary conditions for the acceleration stage. In the acceleration stage, a coupled problem of the quasi-neutral plasma region and the near-wall sheath is considered. Our previously developed model of the acceleration stage [17] is extended to include the effect of sheath expansion. It was shown that the length of the acceleration channel cross section decreases due to sheath expansion [17]. This effect can be explained by invoking the following simple argument. When a negative voltage (cathode potential in the case considered) is applied to a surface immersed in a plasma, electrons are repelled from the surface, leading to sheath formation. Electrons drift away from the 
surface due to the presence of the high electric field. In the steady state, the ions are then accelerated toward the surface by the electric field of the sheath. In the one-dimensional steady-state case the sheath thickness can be estimated according to the Child-Langmuir law $[24,25]$ :

$$
s=\left(\frac{4}{9} \varepsilon\right)^{1 / 2}\left(\frac{2 e}{m_{i}}\right)^{1 / 4} \frac{U^{3 / 4}}{\left(e Z_{i} N_{s} V_{s}\right)^{1 / 2}}
$$

In a bismuth TAL, the channel wall has a potential equal to the cathode potential and therefore the voltage across the sheath $U$ is equal to the plasma potential and varies along the channel. Similarly, the steady-state sheath thickness in a partially magnetized plasma was calculated in a plasma immersion ion implantation system [26]. One can see that the steady-state sheath thickness is determined by the plasma density and ion velocity at the sheath edge for a given bias voltage. In general, the high-voltage sheath expands significantly and the quasi-neutral plasma region is confined in the middle of the channel. For instance, in the case of a $5 \mathrm{kV}$ discharge voltage, the sheath thickness is about several centimeters, which is a significant portion of the channel width. Thus, sheath expansion leads to a decrease of the acceleration region length.

The typical plasma density distribution in the acceleration channel is shown in Fig. 5. In these calculations, electron depletion due to the high-voltage sheath expansion is taken into account. The plasmasheath interface is considered as a boundary for the quasi-neutral plasma flow. One can see that the quasi-neutral plasma region is confined in the middle of the channel especially at the anode side of the channel.

One of the important parameters of the acceleration region is the acceleration layer length. Previously, it was shown that the acceleration layer length decreases due to the effect of the sheath near the wall [17]. This effect can be explained using rather simple physical reasoning. The acceleration layer length is determined by the balance between the electron flux and the electron drift across the magnetic field:

$$
e \mu_{\perp} n_{e} E=j_{e}=\frac{I}{A(s)}
$$

where $A(s)$ is the acceleration channel cross section dependent on the sheath thickness $s$. The electric field can be estimated as $U_{a} / d_{a}$. Taking this into account and assuming the Bohm-type electron mobility across the magnetic field $\mu_{\perp}=1 /(\alpha B)$ one can obtain

$$
d_{a} \sim r_{c e} A(s)
$$

Thus, one can see that the acceleration layer length decreases with $A(s)$ decrease due to sheath expansion. It should be pointed out that sheath expansion should be taken into account if one needs to calculate the acceleration layer length for design of a new TAL.

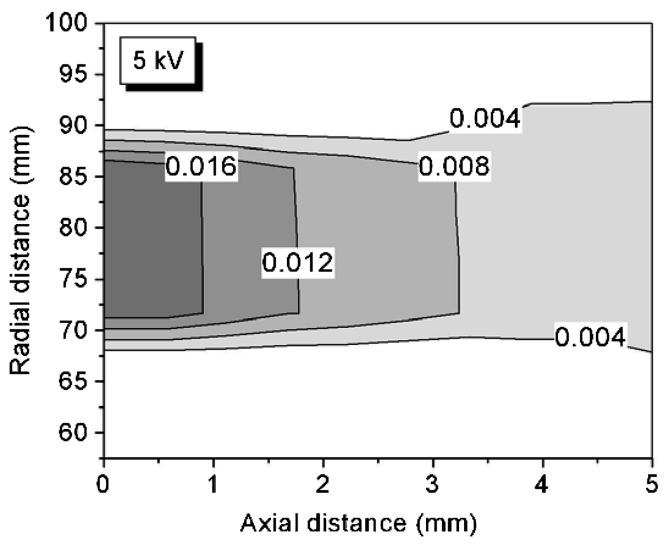

Fig. 5 Electron density distribution in the acceleration channel. The wall has the cathode potential. Electrons are depleted from the sheath due to a large potential drop.

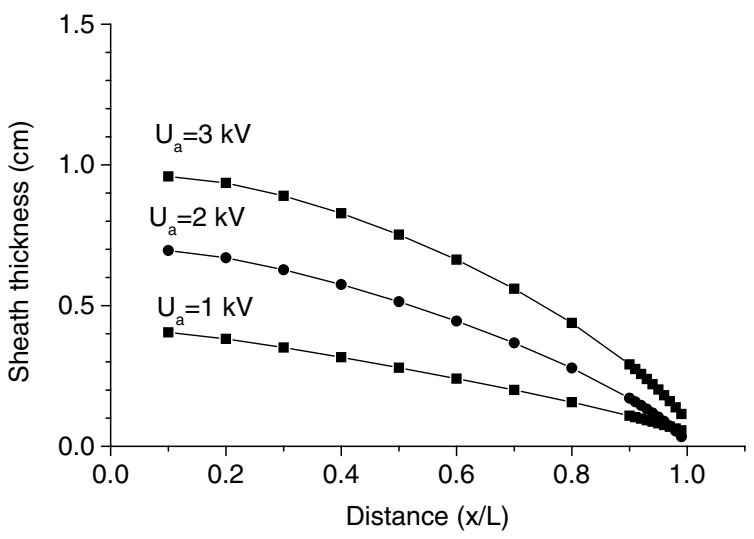

Fig. 6 Sheath thickness with acceleration voltage as a parameter.

\section{Erosion Study}

Based on the output from the model, we calculated the erosion of the guard ring, which is the most critical part of the second stage of the considered TAL. Unfortunately, there are no available measured data on sputtering coefficients for many materials under bismuth bombardment. Therefore we use a simulated sputtering yield of bismuth ions on a molybdenum target predicted by the TRIM code. $\underline{\S}$ We consider guard ring walls made out of molybdenum in order to compare model predictions with some available experimental data summarized in [16]. The sputtering yield depends on the ion energy and angle of incidence. Because of sheath expansion in the acceleration stage as considered in the previous section, ions accelerate to the wall across the sheath to the energy equivalent of the sheath potential drop. This adds to the ion energy due to acceleration in the electric field in the axial direction. Toward the exit plane, the potential drop across the sheath decreases; thus the ion velocity component normal to the wall also decreases. The sheath thickness distribution along the acceleration channel and dependence on the acceleration voltage are shown in Fig. 6. It can be seen that the sheath thickness significantly decreases toward the thruster exit plane. This effect will be important in the evaluation of the erosion profile as will be described later.

The calculated erosion rate is shown in Fig. 7. One can see that erosion peaks inside the channel near the exit plane. The erosion rate then decreases toward the exit plane due to the decrease of the potential drop in the radial direction in the sheath. On the other hand, the erosion rate profile depends on the fraction of the voltage inside the channel as shown in Fig. 7. In the case of an entire voltage drop inside the channel, the erosion rate peaks near the exit plane, whereas in the case of a significant voltage fraction in the near plume, the erosion profile has its peak at the exit plane. The later dependence is more consistent with the experimental observations [16]. One can see that about $1 \mathrm{~mm}$ will be eroded after $260 \mathrm{~h}$, which corresponds to experimental data [16]. It is known that in general significant ion acceleration occurs in the near plume, thus suggesting that one should consider only a fraction of the acceleration voltage inside the channel. It should be pointed out that the overall erosion profile and the total erosion of the guard ring wall are consistent with experimental observations [16].

\section{Plume Study}

2-D plume modeling is also being conducted for spacecraft integration assessment. Some preliminary results are presented here. The number density at the exit of the thruster is of the order of $10^{16}-10^{18} \mathrm{~m}^{-3}$. This indicates that the flow is not in the hydrodynamic regime, but also that there will be some collisions that need to be taken into account. The numerical approach employed in the present work employs a hybrid particle-fluid approach. The bismuth ions (only singly charged) and atoms are simulated as

\footnotetext{
${ }^{\S}$ Yalin, A., private communication, 2005.
} 


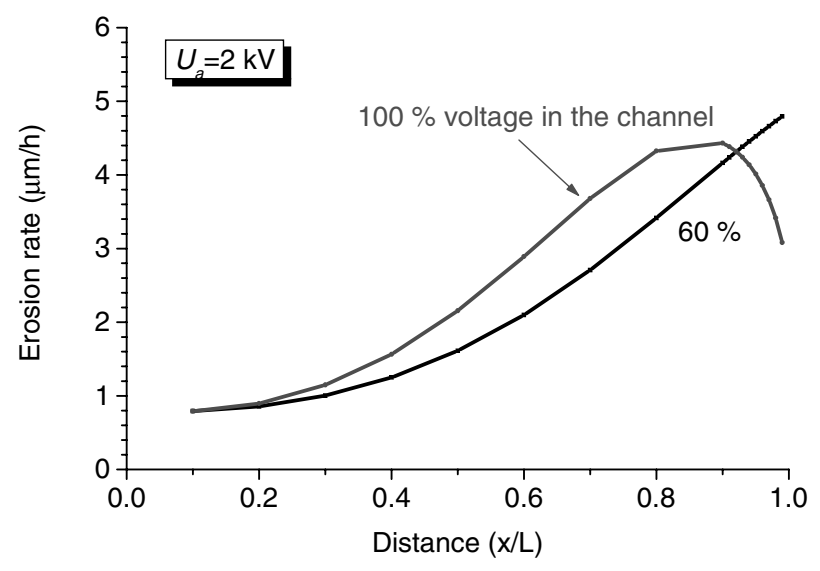

Fig. 7 Erosion rate distribution with the voltage drop inside the acceleration channel as a parameter. Mass flow rate $=10 \mathrm{mg} / \mathrm{s}$, $I_{a}=5 \mathrm{~A}, U_{a}=2 \mathrm{kV}$.

particles using the direct simulation Monte Carlo (DSMC) method [27] to simulate collisions, and the particle-in-cell (PIC) method [28] to accelerate the ions self-consistently in electrostatic fields. Because of their significantly smaller length and time scales, electrons are simulated using a hydrodynamic fluid approach. In the present work, the electron fluid model simply uses the Boltzmann relation that provides the plasma potential based on the plasma number density that is obtained directly from the spatial distribution of the ions under the assumption of charge neutrality. Although more advanced electron fluid models have been developed recently for xenon plasma plumes [29], the Boltzmann relation is considered a sufficient approach for this preliminary study. A critical aspect of these simulations concerns the cross sections for charge and momentum exchange as these are important mechanisms leading to plume divergence. In the present work, the charge exchange cross sections employ the semiempirical general model of Sakabe and Isawa [30]. When a charge exchange event occurs between a bismuth ion and a bismuth atom, it is assumed that no collisional scattering occurs. Based on conclusions drawn for an xenon plasma [31], it is assumed that the momentum exchange cross section between an ion and an atom is identical to that for charge exchange. For momentum exchange between two atoms, the variable hard sphere (VHS) collision model of Bird [27] is employed. Coulomb collisions are omitted from the present analysis. All momentum exchange collisions are assumed to follow isotropic scattering.

Most of the properties at the thruster exit are obtained from the thruster modeling. The ion velocity distribution function is a shifted Maxwellian with drift velocity, and ion density is taken from the 2-D model of the acceleration stage. The initial plume angle is then allowed to vary linearly across the radial extent of the thruster exit between lower and upper values. The ion temperatures are varied in order to obtain the best agreement with the available experimental data. $R=0$ is applied as an axis of rotation so $R<0$ is not included in simulations. In both experiment and simulation, the ion collecting surface was perpendicular to the thruster axis. The plasma potential in the thruster exit is assumed to be $0 \mathrm{~V}$ and the electron temperature in the Boltzmann model is $15 \mathrm{eV}$. In Figs. $8 \mathrm{a}$ and $8 \mathrm{~b}$, contours of the ion and atom number densities are shown, respectively. The simulations are conducted at a finite back pressure of $10^{-5}$ torr. These results clearly indicate the very focused beam that is produced by the VHITAL thruster. Indications of charge exchange ions escaping from the main beam are also present. Very limited experimental data in the plume are available from the Russian experiments of the 1980s [16]. The most reliable data consist of radial profiles of ion current density taken $70 \mathrm{~cm}$ downstream of the thruster exit. Figures $\underline{9}$ and 10 show a comparison between these measured data and the simulation data obtained with a thruster exit ion temperature of $1 \mathrm{eV}$. Initial angle $\alpha$ indicates the lower and upper values of the initial plume angle at the thruster exit assumed for each plume simulation. In the special case of $\alpha=+4,+4$ this means that a uniform plume angle of $4 \mathrm{deg}$ was applied, and so it is like a beam skewed to an angle of $4 \mathrm{deg}$.

The simulation results offer generally good agreement with the measured data, but are sensitive to the values assumed at the thruster exit plane for the ion temperature and initial plume angle $\alpha$ as shown in Figs. 9 and 10. One can see that ion current density distribution is affected strongly by the plume angle at the thruster channel exit plane. A smaller initial angle leads to a more collimated plume. Future plume simulation work will consider additional experimental data sets as well as working toward the inclusion of magnetic field effects on the plume plasma.

\section{Concluding Remarks}

In this paper, a model of a two-stage thruster with anode layer based on a two-dimensional hydrodynamic approach was described. We considered both ionization and acceleration stages separately, having used the solution of the first stage as the boundary conditions for the second stage. It was found that the predicted current-voltage characteristic of the discharge in the first stage is in good agreement with experiment. The thruster has an optimal discharge characteristic in terms of fairly constant acceleration current in a narrow range of discharge voltage (90-250 V). Higher discharge voltage (above $250 \mathrm{~V}$ ) leads to very high electron temperature in the first stage and causes significant increase of the discharge current as well as the acceleration stage current. In the acceleration channel, plasma-wall interactions such as formation of the near-wall sheath was

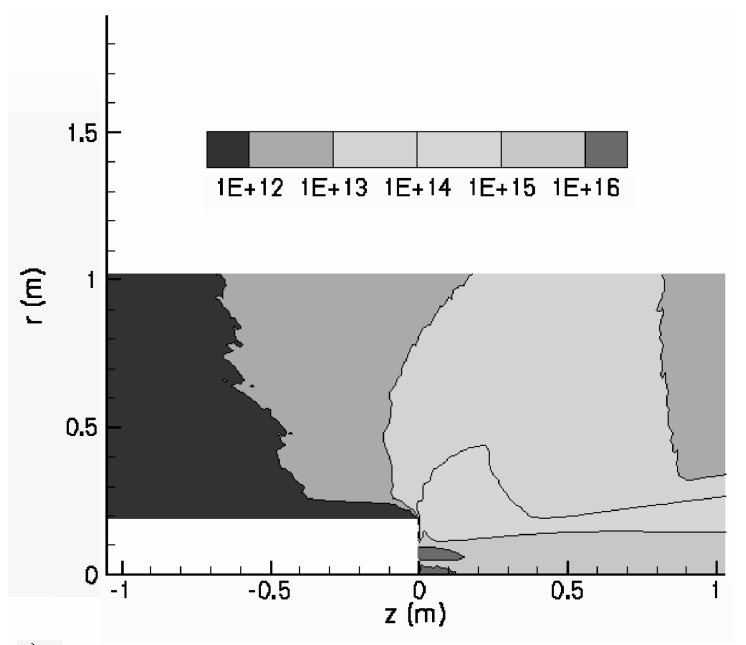

a)

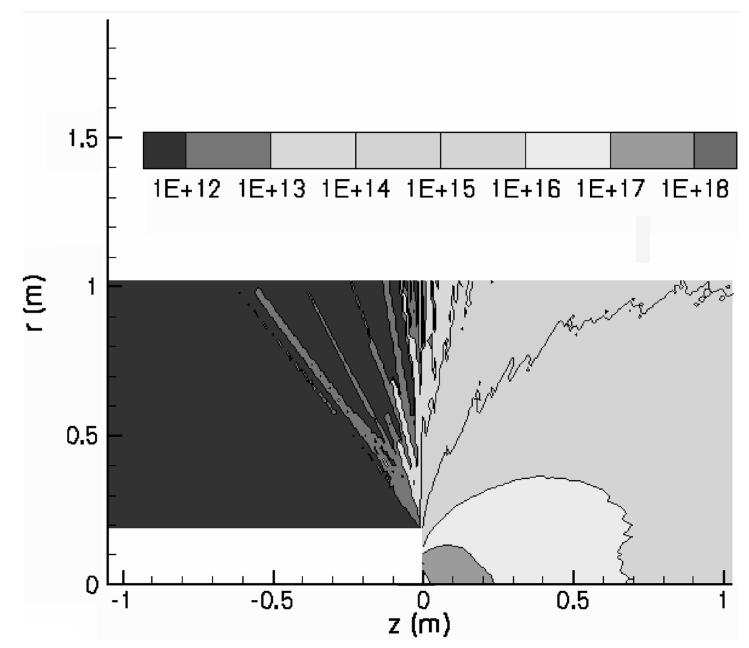

b)

Fig. 8 Number density $\left(\mathrm{m}^{-3}\right)$ contours in the VHITAL plume for a) ions and b) atoms. 


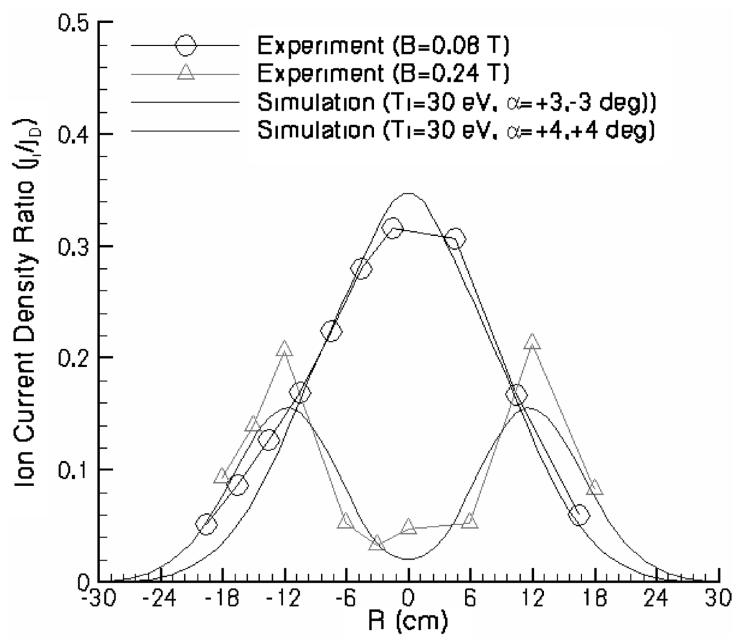

Fig. 9 Radial profile of ion current density at $70 \mathrm{~cm}$ from the thruster exit. Effect of the magnetic field and initial angle. Initial angle $\alpha$ indicates the lower and upper values of the initial plume angle at the thruster exit assumed for each plume simulation.

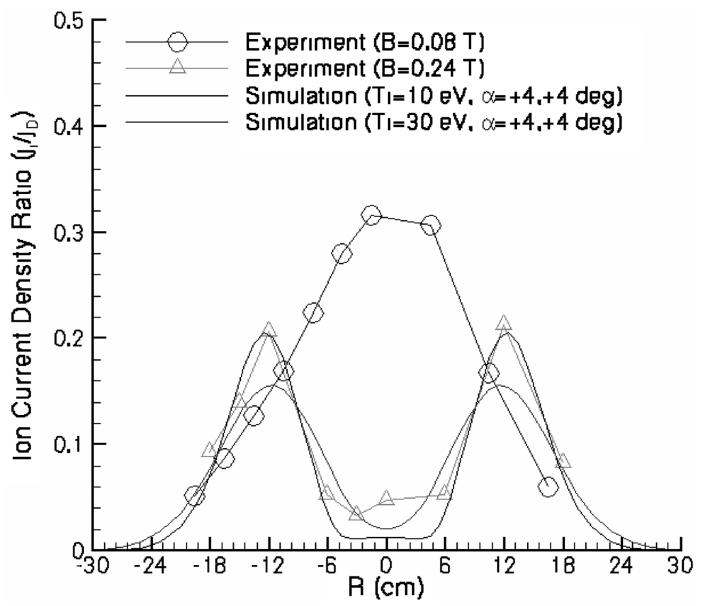

Fig. 10 Radial profile of ion current density at $70 \mathrm{~cm}$ from the thruster exit. Effect of the magnetic field and initial ion temperature. Initial angle $\alpha$ indicates the lower and upper values of the initial plume angle at the thruster exit assumed for each plume simulation.

considered. An expansion of the high-voltage sheath near the acceleration channel wall was investigated. The model predicted that under the typical conditions, the near-wall sheath expands significantly and the quasi-neutral plasma region is confined in the middle of the channel. For instance, in the case of a $3 \mathrm{kV}$ discharge voltage, the sheath thickness is about $1 \mathrm{~cm}$, which is a significant portion of the channel width. In addition it was shown that near-wall sheath expansion leads to a shorter acceleration region.

Based on the plasma properties in the acceleration region, the guard ring erosion profile was calculated and it was shown that the erosion rate profile depends on the fraction of the voltage inside the channel. In the case of an entire voltage drop inside the channel, the erosion rate peaks near the exit plane, whereas in the case of a significant voltage fraction in the near plume, the erosion profile has its peak at the exit plane. The erosion profile and the total erosion of the guard ring wall were found to be consistent with experimental observations.

Plasma flow analysis provided the boundary conditions for the plume expansion study. A hybrid particle-fluid model of the thruster plume was developed. It was shown that the ion current density distribution in the plume generally agrees well with available experimental data thus partially validating our overall modeling approach.

\section{Acknowledgments}

The research was funded by NASA's Exploration Systems Missions Directorate, managed by John Warren, Associate Director for Advanced Systems and Technology, Prometheus Nuclear Systems and Technology Program. The authors thank A. Yalin for providing sputtering coefficients used in these simulations and $\mathrm{C}$. Marrese-Reading, A. Sengupta, M. Cappelli, and A. Semenkin for valuable discussions.

\section{References}

[1] Yushmanov, E. E., "Radial Distribution of the Potential in Cylindrical Trap with Magnetron Ion Injection," Plasma Physics and Problem of Controlled Fusion, edited by M. A. Leontovich, Vol. 4, USSR Academy of Science, Moscow, 1958, pp. 235-237 (in Russian).

[2] Zharinov, A. V., "Electric Double Layer in Strong Magnetic Field," Kurchatov Institute Report, 1961 (in Russian).

[3] Seikel, G. R., and Reshotko, E., "Hall Current Ion Accelerator," Bulletin of the Americal Physical Society, Ser. II, Vol. 7, 1962, p. 414.

[4] Salz, F., Meyerand, R. G., and Lary, E. C., "Ion Acceleration in a GyroDominated Neutral Plasma-Experiment," Bulletin of the Americal Physical Society, Ser. II, Vol. 7, 1962, p. 441.

[5] Salz, F., Meyerand, R. G., and Lary, E. C., "Ion Acceleration in a GyroDominated Neutral Plasma-Theory," Bulletin of the Americal Physical Society, Ser. II, Vol. 7, 1962, p. 441.

[6] Janes, G. S., and Lowder, R. S., "Anomalous Electron Diffusion and Ion Acceleration in a Low-Density Plasma," Physics of Fluids, Vol. 9, No. 6, 1966, pp. 1115-1123.

[7] Zharinov, A. V., and Popov, Yu. S., "Acceleration of Plasma by a Closed Hall Current," Soviet Physics-Technical Physics, Vol. 12, No. 2, 1967, pp. 208-211.

[8] Morozov, A. I., "Effect of Near-Wall Conductivity in Magnetized Plasma," Journal Applied Mathematics-Technical Physics, Vol. 3, 1968, pp. 19-22.

[9] Zhurin, V. V., Kaufman, H. R., and Robinson, R. S., "Physics of Closed Drift Thrusters," Plasma Sources Science \& Technology, Vol. 8, No. 1, 1999, pp. R1-R20.

[10] Morozov, A. I., and Savelyev, V. V., in Review of Plasma Physics, edited by B. B. Kadomtsev, and Shafranov, V. D., Vol. 21, Consultant Bureau, New York, 2000, p. 203.

[11] Jacobson, D. T., Jankovsky, R. S., Rawlin, V. K., and Manzella, D. H., "High-Voltage TAL Performance," AIAA Paper 2001-3777, July 2001.

[12] Tverdokhlebov, S., Semenkin, A., and Polk, J., "Bismuth Propellant Option for Very High Power TAL Thruster," AIAA Paper 2002-0348, Jan. 2002.

[13] Marrese-Reading, C., Sengupta, A., Frisbee, R., Polk, J., Cappelli, M., Boyd, I., Keidar, M., Tverdokhlebov, S., Semenkin, S., Markusic, T., Yalin, A., and Knowles, T., "The VHITAL Program to Demonstrate the Performance and Lifetime of a Bismuth-Fueled Very High Isp Hall Thruster," AIAA Paper 05-4564, July 2005.

[14] Kieckhafer, A., and King, L., "Energetics of Propellant Options for High-Power Hall Thrusters," AIAA Paper 2005-4228, July 2005.

[15] Soloduchin, A. E., and Semenkin, A. V., "Study of Discharge Channel Erosion in Multi-Mode Anode Layer Thruster," Proceedings of the 28th International Conference on Electric Propulsion, Electric Rocket Propulsion Society, Worthington, OH, 2003; also IEPC Paper 20030204.

[16] Erofeev, V. E., and Leskov, L. V., "Hall Accelerator with Anode Layer," Physics and Application of Plasma Accelerators, edited by A. I. Morozov, Nauka, Minsk, 1974, pp. 18-47 (in Russian).

[17] Keidar, M., Boyd, I. D., and Beilis, I. I., "Modeling of a High-Power Thruster with Anode Layer," Physics of Plasmas, Vol. 11, No. 4, 2004, pp. 1715-1722.

[18] Keidar, M., Boyd, I. D., and Beilis, I. I., "Plasma Flow and Plasma-Wall Transition in Hall Thruster Channel," Physics of Plasmas, Vol. 8, No. 12, 2001, pp. 5315-5322.

[19] Freund, R. S., Wetzel, R. C., Shul, R. J., and Hayes, T. R., "CrossSection Measurements for Electron-Impact Ionization of Atoms," Physical Review A, Vol. 41, No. 7, 1990, pp. 3575-3595.

[20] Keidar, M., and Beilis, I. I., "Electron Transport Phenomena in Plasma Devices with ExB Drift," IEEE Transactions on Plasma Science, Vol. 34, No. 4, 2006.

[21] Bohm, O., The Characteristics of Electrical Discharges in Magnetic Fields, edited by A. Guthrue, and R. K. Wakerling, McGraw-Hill, New York, 1949. 
[22] Keidar, M., Beilis, I., Boxman, R. L., and Goldsmith, S., "2D Expansion of the Low-Density Interelectrode Vacuum Arc Plasma Jet in an Axial Magnetic Field," Journal of Physics D: Applied Physics, Vol. 29, 1996, pp. 1973-1983.

[23] Popov, Yu. S., "Penning Low-Pressure Discharge with Cold Cathode," Soviet Physics-Technical Physics, Vol. 37, No. 1, 1967, pp. 118-126.

[24] Child, C. D., "Discharge from Hot CaO," Physical Review, Vol. 32, 1911, p. 492.

[25] Langmuir, I., "The Effect of Space Charge and Residual Gases on Thermionic Currents in High Vacuum," Physical Review, Ser. II, Vol. 2, 1913, p. 450.

[26] Keidar, M., Monteiro, O. R., Anders, A., and Boyd, I. D., "Magnetic Field Effect on the Sheath Thickness in Plasma Immersion Ion Implantation," Applied Physics Letters, Vol. 81, No. 7, 2002, pp. 11831185.

[27] Bird, G. A., Molecular Gas Dynamics and the Direct Simulation of Gas
Flows, Oxford Univ. Press, Oxford, England, U.K., 1994.

[28] Birdsall, G. A., and Langdon, B., Plasma Physics Via Particle Simulation, Adam Hilger, Bristol, England, U.K., 1994.

[29] Boyd, I. D., and Yim, J. T., "Modeling of the Near-Field Plume of a Hall Thruster," Journal of Applied Physics, Vol. 95, No. 9, 2004, pp. 45754584.

[30] Sakabe, A., and Izawa, Y., "Simple Formula for the Cross Sections of Resonant Charge Transfer Between Atoms and Their Positive Ions at Low Impact Velocity," Physical Review A, Vol. 45, No. 3, 1992 , pp. 2086-2089.

[31] Boyd, I. D., and Dressler, R. A., "Far-Field Modeling of the Plasma Plume of a Hall Thruster," Journal of Applied Physics, Vol. 92, No. 4, 2002, pp. 1764-1774.
E. Choueiri Associate Editor 